Press, New York. - 70. Riddle, M. C., J. Clin. Invest. 8, 69 (1930). - 71. Opsaht, R., Acta med. Scand. 102, 611 (1939). 72. Patridge, R. E. H. und J. J. R. Duthie, Brit. Med. J. 1, 89 (1963). - 73. TAYlor, K. B., Lancet 2, 106 (1959). - 74. FreiREICH, E. J., J. F. Ross, T. B. BAYLES, C. P. Emerson und S. C. FinCH, J. Clin. Invest. 36, 1043 (1957). - 75. Lewrs, S. M. und I. H. Porter, Ann. Rheumat. Dis. 19, 54 (1960). - 76. PABENBerG, K., Klin. Wschr. 39, 522 (1961). - 77. Mandel, P. und P. Chambon, Bull. Soc. Chim. biol. 41, 989 (1959). - 78. SChweiger, H. G., S. Rapaport und E. Schölzel, Hoppe-Seyler's Z. physiol. Chem. 313, 97 (1958). - 79. SCHweiger, H. G., Int. Rev. Cytol. 13, 135 (1962). - 80. Schweiger, H. G., H. J. Bremer und E. Schweiger, Hoppe-Seyler's Z. physiol. Chem. 332, 17 (1963). - 81. Carlström, B., O. Lövgren und B. Sjörgen, Ann. Rheumat. Dis. 8, 293 (1949). - 82. Carlström, B., O. Lövgren und B. Syörgen, Acta Med. Scand. 110, 230 (1942). 83. Carlström, B., O. Lövgren und B. Sjörgen, Acta Med. Scand. 115, 568 (1943). - 84. CArLström, B., O. Lövgren und B. SJörgen, Klin. Wschr. 20, 793 (1941). - 85. LeHNINGER, A. L., J. biol. Chemistry 54, 309 (1944). - 86. Schmrd, J., Med. klin. Wschr. f. Klinik u. Praxis 48 (1953) Nr. 36. - 87. Vogr, M., J. Physiol. 108, 45 (1949). - 88. STONER, H. B. und H. N. GreEN, Brit. Exper. Path. 31, 603 (1950). - 89. Ruskin, S. L., Amer. J. Digest. Dis. 13, 311 (1946). - 90. Netrelbladt, E. und B. M. SANDELl, Ann. theum. Dis. London, 22, 269 (1963). - 91. Elliot, W. H. und B. M. SANDEll, J. biol. Chemistry 201, 661 (1953). - 92. GyörkI, J. und B. M. SANDell, Nord. med. 62, 1788 (1959). - 93. Rapoport, S., G. M. Guest und M. WING, Proc. Soc. exp. Biol. Med. 57, 344 (1944). - 94. Hofmann, E. G. G. und S. Rapoport, Hoppe-Seyler's Z. physiol. Chem. 304, 157 (1956). - 95. Löhr, G. W., H. D. Waller, O. Karges, B. Schlegel und A. A. MülleR, Klin. Wschr. 36, 1008 (1958). 96. Rapoport, S., J. Clin. Invest. 26, 591 (1947). - 97. Gabrio, B. W., D. M. Donohue und C. A. Finch, J. Clin. Invest. 34, 1509 (1955). - 98. Jorgensen, S., Acta pharmac. tox., K'hrn 13. 102 (1957). - 99. KöLLE, G., Zschr. Rheumaforsch. 21, 185
(1962). - 100. Feigelson, P. H. und M. Fergelson, J. biol. Chemistry 238, 1073 (1963). - 101. Gerlach, E., A. Fleckenstein und K. J. Freundt, Pflügers Arch. ges. Physiol. Menschen, Tiere, 263, 682 (1957). - 102. Bishop, C., J. biol. Chemistry 236, 1778 (1961). - 103. ZIMMERManN, E. E. und B. Magasanik, J. biol. Chemistry 239, 293 (1964). - 104. MAGER, J. und B. Magasanik, J. biol. Chemistry 235, 1474 (1960). - 105. Guarino, A. J. und G. Yüregier, Biochim. biophysics Acta (Amsterdam) 36, 157 (1959). - 106. Brwas, B. B. und R. Abrams, Arch. Biochem. Biophysics 92, 507 (1961). - 107. Lowy, B. A. und E. R. JAFFe, G. A. VANDerhoff und I. M. London, J. biol. Chemistry 230, 409 (1958). - 108. Conway, E. J. und R. Cooke, Biochem. J. 33, 479 (1939). - 109. MrLlis, G. C. und L. B. Summers, Arch. Biochem. Biophysics 84, 7 (1959). - 110. HuenNekens, F. M., E. Nurk und B. W. Gabrio, J. biol. Chemistry 221, 971 (1956). - 111. Sandberg, A., und G. R. Lee, Cartwrigit, G. E. und M. M. Wintrobe, J. Clin. Invest. 34, 1823 (1955). - 112. Rubenstein, D. und O. F. Denstadt, Canad. J. Biochem. Physiol. 34, 927 (1956). - 113. Liebermann, I., J. Amer. chem. Soc. 78, 251 (1956). - 114. Braunstein, A. E. und G. I. Vilenkina, Biochima 23, 887 (1958). - 115. Henderson, J. F. und G. A. Le PAge, J. biol. Chemistry 234, 3219 (1959). - 116. LAJTha, L. G. und J. R. VANE, Nature (London) 182, 191 (1958). - 117. Theil, E. C. und St. ZanNENHof, J. biol. Chemistry 238, 3058 (1963). - 118. Dunn, D. B. und J. D. SMrth, Biochem. J. 68, 627 (1958). - 119. Dunn, D. B., J. D. SMrth und P. F. SpaHR, J. molecular Biol. 2, 113 (1960). - 120. LittleFIELD, J. W. und D. B. DunN, Biochem. J. 70, 642 (1958). 121. Haddox, C. H. jr. und M. S. SASLAw, J. Clin. Invest. 42 , 435 (1963). - 122. Betr, J. M., Ann. rheumat. Dis., London 21, 63 (1962). - 123. Spiera, H., Arthr. Rheum. 6, 364 (1963). 124. Dion, H. W., D. G. Calkins und J. J. Pfiffner, J. Amer. chem. Soc. 76, 948 (1954). - 125. BRown, F. B., J. C. CaIN, D. E. GaNt, L. F. J. Parker und E. L. SMITH, Biochem. J. 59, 82 (1955).
Dozent Dr. med. J. Schmid Wien XIX, Österreich Scheibengasse 13

\title{
Schnellmethode zur Darstellung von Fettsäuremethylestern für die gaschromatographische Analyse
}

\author{
Von M. Doss und K. Oetre \\ Aus dem Pbysiologiscb=Chemischen Institut der Universität Köln (Direktor: Prof. Dr. Dr. h. c. E. Klenk)
}

(Eingegangen am 13. August 1964)

\begin{abstract}
Für die gaschromatographische Analyse wird eine quantitative Mikromethode beschrieben, die die Herstellung von Methylestern aus Glyceriden, Glycerinphosphatiden und Cholesterinestern durch Umesterung mit Natriummethylat innerhalb von fünf Minuten gewährleistet.
\end{abstract}

Micro quantities of methyl esters for gas chromatography can be prepared quantitatively in five minutes from glycerides, glycerophosphatides and cholesterol esters by transesterification with sodium methylate.

Fettsäuren werden im allgemeinen als Methylester gaschromatographisch analysiert, zu deren Darstellung Veresterungs- und Umesterungsmethoden zur Verfügung stehen. Zur Veresterung von freien Fettsäuren sind folgende Verfahren beschrieben worden:

1. Die säure-katalysierte Veresterung mit Methanol- $\mathrm{HCl}$ für langkettige (1) und mit 2-Chloräthanol- $\mathrm{HCl}$ für kurzkettige Fettsäuren (2).
2. Die Veresterung mit Diazomethan (3).

3. Die Veresterung mit Methanol-Bortrifluorid (4).

Für die Uimesterung verwendet man vorwiegend Methanol$\mathrm{HCl}\left(\mathrm{H}_{2} \mathrm{SO}_{4}\right)$. Daneben sind alkali-katalysierte Methoden geübt worden (5-9).

Im Rahmen von Untersuchungen an Sphingolipoiden (10) zeigte sich, daß esterartig verknüpfte Fettsäuren rasch mit $0,1 n$ methanolischer $\mathrm{NaOH}$ als Methylester 
abgespalten wurden. Die Schnelligkeit der Reaktion veranlaßte uns, die alkali-katalysierte Umesterung auf ihre Brauchbarkeit als Routinemethode für die Gaschromatographie zu prüfen. Man erwartet von einer derartigen Methode eine schnelle und quantitative Umsetzung mit möglichst geringem apparativen Aufwand.

Die alkali-katalysierte Umesterung mit Na-Methylat oder methanolischer $\mathrm{NaOH}$ ist eine bekannte Reaktion. Der Ester existiert in zwei mesomeren Grenzformen, deren labilere sich durch Aufrichtung der $\mathrm{C}=\mathrm{O}$-Doppelbindung bei gleichzeitigem Auftreten einer Elektronenlücke am zugehörigen C-Atom auszeichnet (Formel 1). Vermutlich wird die Ausbildung der labileren Form des Esters durch Alkali begünstigt, wenn auch nicht in dem Ausmaß wie bei der säure-katalytischen Umesterung. Das Alkali katalysiert durch Förderung der Bildung der nucleophilen $\mathrm{CH}_{3} \mathrm{O}^{-}$-Gruppe deren Anlagerung an das positiv geladene C-Atom des Esters. Der entstandene Komplex setzt sich infolge des großen Überschusses an Methylationen schnell im Sinne der Reaktion (2) um.

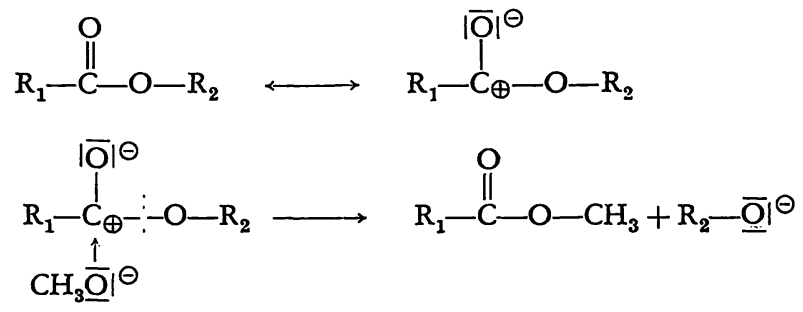

Die alkali-katalysierte Umesterung wurde bereits von KLENK und ReNnKamp (5) im Jahre 1945 zur Abtrennung einer der Rohsphingomyelinfraktion beigemischten Glycerinphosphatid-Verunreinigung benutzt, wobei sich esterartig gebundene Fettsäuren als Äthylester abspalteten. KuemMEL (6) beschrieb 1958 die Umesterung von Fetten und Ölen durch methanolische $\mathrm{NaOH}$. Die Anwendung von Natriummethylat zur Gewinnung von Methylestern für die gaschromatographische Analyse wurde von LUDDY und Mitarbeitern (7) 1960 mitgeteilt. Infast unveränderter Weise fand dieses Verfahren weitere Verbreitung $(8,9)$. Jedoch bietet die von LuDDY beschriebene Art der Anwendung gegenüber der Umesterung mit Methanol- $\mathrm{HCl}$ keinen entscheidenden Vorteil mit Ausnahme der Umesterung von Triglyceriden. Unsere Versuche ergaben, daß sich die Reaktionszeit der Umesterung für alle in Frage kommenden Lipoide erheblich verkürzen läßt, was eine Vereinfachung des apparativen Aufwandes mit sich bringt. Deshalb eignet sich das neue Verfahren speziell für die Gaschromatographie.

\section{Methodik}

\section{Reagenzien:}

$0,5 n$ Na-Methylat (Stammlösung von $1,15 \mathrm{~g}$ metallischen Natriums in $100 \mathrm{~m} l$ Methanol p. a. unter Kühlung); $0,5 n$ wäßrige Salzsäure; Petroläther (Kp. 30-600)/Diäthyläther 9:1 (V/V); Chloroform; Benzol; Natriumsulfat.
Apparatur $\left.)^{1}\right):$

Ein Reaktionsgefäß (Normalschliff 19/26) mit Kühlfingereinsatz; Maße und Ausführung siehe Abbildung 1. Das Reaktionsgefäß wird nach der Umesterung mit Stopfen als Extraktionsgefäß verwendet.

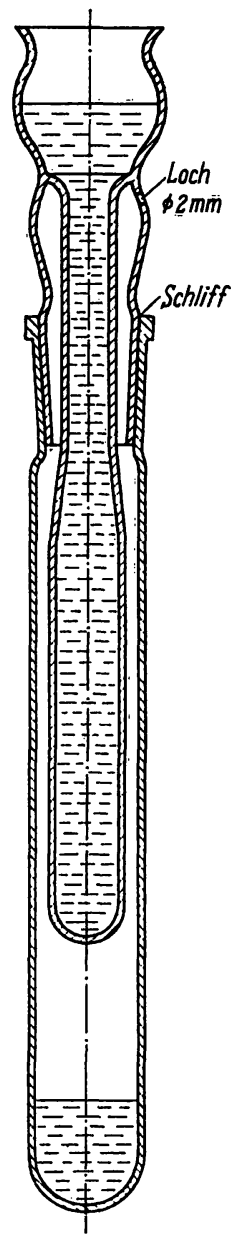

Abb. 1

Umesterungs-Apparatur $\left.{ }^{1}\right)$ : Reaktions- und Extraktionsgefäß mit Kühlfingereinsatz; Maßstab 1:2

\section{Ausführung}

A) Umesterung von Gemischen aus Glyceriden, Glycerinphosphatiden und Cholesterinestern oder reinen Cholesterinestern

j-10 mg Substanz, bei kleineren Mengen von Colesterinestern im Gemisch bis $15 \mathrm{mg}$, werden in das Reaktionsgefä $\beta$ der Abbildung 1 eingeführt; $f \in$ lls in Lösung einpipettiert wurde, wird das Lösungsmittel durch $\mathrm{N}_{2}$-Strom abgeblasen; Substanz wird in $0,4 \mathrm{ml}$ Chloroform oder Benzol sorgfältig am Boden gelöst und mit $4 \mathrm{ml} 0,5 n \mathrm{Na}-$ Methylat versetzt. Eine eventuelle Ausfällung von Lipoid geht in der Hitze wieder in Lösung. Nach Zugabe eines Siedesteines und Einsetzen des mit kaltem Wasser gefüllten Kühlfingers läßt man die Probe $5 \mathrm{Min}$. im Olbad von $100^{\circ}$ sieden. Eine Nebenreaktion mit Chloroform, die zur Ausfällung von $\mathrm{NaCl}$ führt, stört die Umesterung nicht. Man kühlt das Gefäß ab, gibt $4 \mathrm{ml} 0,5 n$ wäßrige $\mathrm{HCl}$ und $12 \mathrm{ml}$ Aqua dest. hinzu und schüttelt die Methylester mit $10 \mathrm{~m} l$ Petroläther/Diäthyläther $9: 1 \mathrm{kräftig}$ aus. Die untere wäßrige Phase wird mit einer Saughütchen-Pipette (ausgezogenes Glasrohr) abgezogen und verworfen. Die petrolätherische obere Phase wird in demselben Gefäß zweimal mit $5 \mathrm{ml}$

1) Ernșt Zimmermann, Glastechnische Werkstätten, 5 KölnLindenthal, Joseph-Stelzmann-Str. 52. 


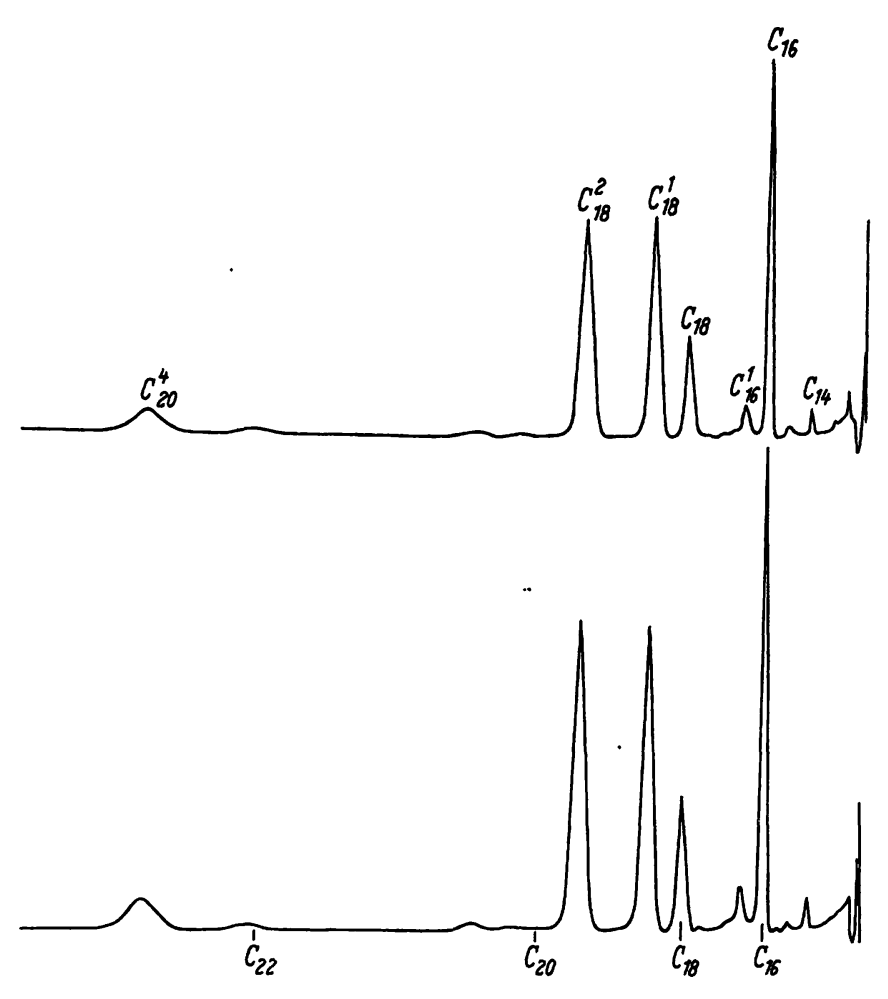

Abb. 2

Gaschromatogramm der Fettsäuremethylester aus Serumlipoiden. Obere Kurve: Umgeestert mit Methanol-6\% HCl. Untere Kurve: Umgeestert mit Na-Methylat. Gaschromatograph der Fa. Pye, Cambridge, England. Säule $134 \times 0,4 \mathrm{~cm}$; Säulenfüllung 20\% EGS auf Kieselgur 0,16-0,20 mm (80-100 mesh); Temperatur $175^{\circ}$; Trägergas Argon; Durchflußgeschwindigkeit $60 \mathrm{~m} / / \mathrm{Min}$; Zellspannung $480 \mathrm{~V}$

Aqua dest. gewaschen und anschließend mit einer kleinen Menge Natriumsulfat getrocknet. Der Methylesterextrakt wird in ein spitz auslaufendes Reagenzglas überführt und das Lösungsmittel im Stickstoffstrom abgedampft. Die Probe ist fertig zur gaschromatographischen Analyse.

B) Umesterung von Glyceriden und/oder Glycerinphosphatiden: $5-20 \mathrm{mg}$ werden in das Reaktionsgefäß eingeführt, in $0,4 \mathrm{ml}$ Chloroform oder Benzol gelöst und mit $4 \mathrm{ml} 0,25 n$ Na-Methylat (Stammlösung 1:1 mit Methanol verdünnt) versetzt. Das Gemisch wird im Reaktionsgefä $ß$ mit Schliffstopfen bei Zimmertemperatur 1-2 Min. kräftig geschüttelt. Dadurch wird das bei Eintragen des Na-Methylats teilweise ausfallende Lipoid unter Umesterung gelöst. Nach Zugabe von $4 \mathrm{ml} 0,5 n$ wäßriger $\mathrm{HCl}$ und $12 \mathrm{~m} l$ Aqua dest. werden die Methylester wie oben beschrieben aufgearbeitet.

Falls größere Mengen freies Cholesterin im Methylestergemisch vorliegen und die Gaschromatographie für Methylester und Steroide mit den gleichen Säulen ausgeführt werden, sollte das Cholesterin durch Mikrosublimation (1) oder Chromatographie an kurzen Kieselsäuresäulen abgetrennt werden (7). Als eine sehr saubere und schnelle Methode hat sich auch die präparative Dünnschichtchromatographie erwiesen. Die Auftrennung wird an Kieselgel- $\mathrm{H}^{1}$ )-Platten, 0,5 mm Schicht= dicke mit Petroläther/Diäthyläther (9:1) (Abb. 3) vorgenommen. Zur weiteren Aufarbeitung hat sich das Schichtabsaugg- und Elutionsgerät ${ }^{2}$ ) von GOLDRICK und Hrrsch (11) bewährt.

1) Fa. E. Merck, Darmstadt.

2) Ernst Zimmermann, Glastechnische Werkstätten, 5 KölnLindenthal, Joseph-Stelzmann-Str. 52.

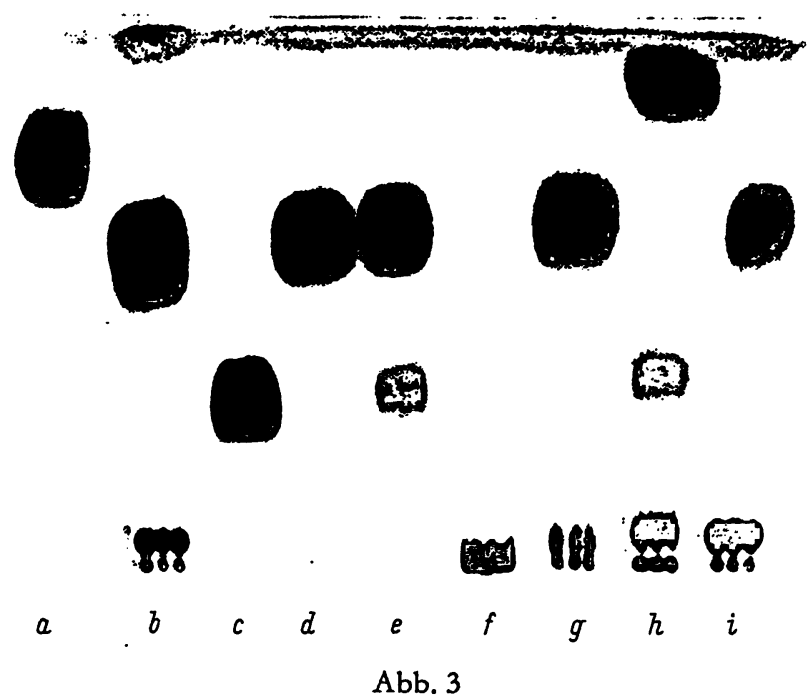

Dünnschichtchromatogramm der mit Na-Methylat zu Methylestern (ME) umgeesterten Lipoide.

Als Test diente ein mit Methanol-HCl umgeestertes ErdnußTriglycerid (TG).

a) Cholesterinester (CE); b) CE-ME; c) TG; d) TG-ME; e) TestME; f) Leberglycerinphosphatid (GP); g) GP-ME; h) Serumlipoide (SL): am Start GP, darüber Cholesterin, TG und CE;

i) SL-ME, in der Nähe des Startpunktes Cholesterin (vgl. b)

Die gaschromatographische Analyse wird in der üblichen Weise durchgeführt, worüber TsснӧрE in dieser Zeitschrift referierte (12).

\section{Dünnschichtchromatographie (13)}

Die dünnschichtchromatographischen Untersuchungen der einzelnen Stoffklassen wurden in vier verschiedenen Lösungsmittelsystemen auf Kieselgel-H-Platten ausgeführt:

1. Petroläther (Kp. 30-60 )/Äthyläther $9: 1$ (V/V) für Cholesterinester, Triglyceride und Methylester (Abb. 3).

2. 1,2-Dichloräthan/Petroläther/Äthyläther $60: 35: 5$ für die Trennung von Dimethylacetalen und Methylestern.

3. Petroläther/Äthyläther/Eisessig 70:28:2 zum Nachweis von freien Fettsäuren.

4. Chloroform/Methanol/Wasser $65: 25: 4$ zum Nachweis von Glycerinphosphatiden, Cerebrosiden und Sulfatiden.

Es liefen jeweils Testsubstanzen mit. Als Sprühreagenz wurde ausschließlich 50-proz. wäßrige Schwefelsäure verwendet. Organische, nicht flüchtige Substanzen stellten sich durch anschließendes Erhitzen der Platten auf $200^{\circ}$ als schwarze Flecke dar (Abb. 3). Die Empfindlichkeit der Sichtbarmachung wurde mit einer Mischung von Cholesterinpalmitat/Stearinsäuremethylester $(1: 100)$ geprüft. Bei $50 \mu \mathrm{g}$ aufgetragener Mischung konnten noch $0,5 \mu \mathrm{g}$ Cholesterinester erkannt werden. Diese Empfindlichkeit ist die Grundlage der in Tabelle 2 angegebenen Werte.

\section{Ergebnisse und Diskussion}

Vergleich der Standardmetboden mit der säure-katalysierten Umesterung

Aus Tabelle 1 und Abbildung 2 geht hervor, dal die quantitativen Daten beider Methoden innerhalb der in der Literatur (14) diskutierten Fehlergrenz liegen. Wir haben, um die Beeinträchtigung einer MethylesterAnalyse durch die Anwesenheit freien Cholesterins zu eruieren, eine Mischung von Triglycerid-Methylestern und Cholesterin im Verhältnis 1:1 chromatographiert. 
Tab. 1.

Vergleich der alkali- mit der säure-katalysierten Umesterung in Flächenprozenten des Gaschromatogramms

\begin{tabular}{|c|c|c|c|c|c|c|}
\hline \multicolumn{3}{|c|}{ Serumlipoid-Methylester } & \multicolumn{4}{|c|}{ Triglycerid-Methylester } \\
\hline $\left.\mathrm{ME}^{1}\right)$ & $\begin{array}{l}\text { Methanol- } \\
6 \% \mathrm{HCl}\end{array}$ & $\begin{array}{c}\text { Na-Methylat } \\
\text { Methode A }\end{array}$ & $\begin{array}{l}\text { Methanol- } \\
6 \% \mathrm{HCl}\end{array}$ & $\begin{array}{c}\text { Na-Methylat } \\
\text { Methode A }\end{array}$ & $\begin{array}{l}\text { Na-Methylat } \\
\text { Methode B }\end{array}$ & $\begin{array}{l}\mathrm{ME}+\text { Chole- } \\
\left.\text { sterin }^{2}\right)(1: 1)\end{array}$ \\
\hline $14: 0$ & 0,85 & 0,75 & - & - & 一 & 一 \\
\hline $16: 0$ & 22,85 & 21,07 & 10,71 & 10,43 & 9,98 & 10,51 \\
\hline $16: 1$ & 1,91 & 2,07 & - & - & - & - \\
\hline $18: 0$ & 9,37 & 9,37 & 3,42 & 4,50 & 3,21 & 3,89 \\
\hline $18: 1$ & 25,72 & 25,97 & 54,22 & 54,01 & 52,44 & 53,98 \\
\hline $18: 2$ & 31,71 & 32,85 & 27,22 & 25,05 & 29,06 & 25,19 \\
\hline $18: 3$ & - & - & 1,42 & 1,55 & 1,71 & 1,96 \\
\hline $20: 0$ & - & - & 1,52 & 1,45 & 1,22 & 1,57 \\
\hline $20: 4$ & 7,60 & 7,81 & - & - & - & - \\
\hline $22: 0$ & - & - & 1,49 & 3,01 & 2,37 & 2,90 \\
\hline
\end{tabular}

1) Anzahl der C-Atome der Fettșäure: Anzahl der Doppelbindungen.

2) Die Beimischung von Cholesterin zum ME zeigte keinen signifikanten Einfluß auf die Analyse (s. Text).

Abkürzung: $\mathrm{ME}=$ Methylester.

Die Proben wurden in Chloroform gelöst injiziert. Das Ergebnis (Tab. 1) zeigte keinen signifikanten Unterschied gegenüber cholesterinfreiem Methylester.

Während durch die säure-katalysierte Überführung in Methylester auch die amidartig gebundenen Säuren (5 Stunden kochen) und die freien Fettsäuren (2 Stunden kochen) verestert und die Acetale in Dimethylacetale umgesetzt werden, zeigte sich in unseren Versuchen mit $\mathrm{Na}-$ Methylat unter den beschriebenen Bedingungen an Cerebrosiden, Acetalen und freien Fettsäuren, daß sowohl die Säureamidgruppe als auch die Acetalbindung intakt bleiben und freie Fettsäuren nicbt verestert werden. Ferner ließen sich Sulfatide mit Na-Methylat nicht spalten. Diese Befunde ergaben sich aus dünnschichtchromatographischen Untersuchungen. Die glatte Umsetzung der mit den beschriebenen Methoden erfaßten Lipoidklassen ist in Abbildung 3 wiedergegeben. Als Testsubstanz dienten Methylester, die aus Triglyceriden durch zweistündiges Kochen mit Methanol- $6 \% \mathrm{HCl}$ dargestellt wurden.

\section{Kritische Aspekte der Umesterungstechnik}

Das Na-Methylat wurde mit nicht nachgetrocknetem Methanol p. a. hergestellt. Der Einfluß kleiner Mengen Wassers auf die Umesterung mit NaMethylat wurde nicht näher untersucht, da die Umsetzung auch in einer methanolischen $\mathrm{NaOH}$ abläuft $(6,10)$. $\mathrm{Da}$ die Alkalikonzentration für die Umesterungsgeschwindigkeit eine Rolle spielt und die Löslichkeit des Na-Methylats in Methanol höher ist als die von $\mathrm{NaOH}$, wurden die Standardmethoden mit Na-Methylat entwickelt.

Voraussetzung für die quantitative Umesterung war die völlige Lösung des eingesetzten Lipoids am Boden des Reaktionsgefäßes vor Zugabe des Alkoholats mittels $0,4 \mathrm{~m} l$ Chloroform oder Benzol. Dies war besonders zu beachten für die Umsetzung von Cholesterinestern. Bei der Umesterung; größerer Lipoidmengen (Tab. 2) kam es zur teilweisen Ausfällung des Lipoids bei Zugabe des Methylats. Die Fällung. löste sich durch Erhitzen oder Schütteln infolge Umsetzung wieder auf.
Es wurden Umesterungen mit $0,1 n, 0,25 n, 0,5 n$ und 1,0 $n \mathrm{Na}$-Methylat vorgenommen. Dabei ergab sich, da $B$ sowohl hinsichtlich der Alkoholatkonzentration als auch in bezug auf die umzusetzende Substanzmenge die Cholesterinester die kritische Lipoidklasse sind. Um auch für die Cholesterinester eine vollständige Umesterung in kürzester Zeit zu erreichen, haben wir für diese Lipoidklasse das Verhältnis von Alkoholat und Substanz auf $5-10 \mathrm{mg} / 4 \mathrm{~m} l \quad 0,5 n \mathrm{Na}$-Methylat bzw. $10-20 \mathrm{mg} / 4 \mathrm{~m} l \quad 1 n \mathrm{Na}$-Methylat (Tab. 2) festgelegt. Die Umesterung wurde allerdings getestet bis zu $40 \mathrm{mg}$ Triglycerid/4 $\mathrm{ml} \quad 0,5 n$ Na-Methylat. Mit Triglyceriden und Glycerinphosphatiden konnte die Umesterung genausogut bei Zimmertemperatur mit $0,25 n$ und $0,5 n \mathrm{Na}$-Methylat vorgenommen werden (Tab. 2). Dabei vollzog sich die Umesterung durch intensives Schütteln des Reaktionsgefäßes innethalb von $1-2 \mathrm{Mi}$ nuten. Bei den Glycerinphosphatiden in Mengen von übèr $15 \mathrm{mg}$ blieb nach dem Schütteln trotz vollständiger Umesterung eine leichte Trübung bestehen. Die Umesterung von Cholesterinpalmitat gelang nur durch kochen. Es war aber möglich, kleinere Mengen Cholesterinesters, z. B. im Serumlipoidgemisch, schon bei Zimmertemperatur umzuestern.

Mit Chloroform als Lösungsmittel an Stelle von Benzol ging die Umesterung in der Hitze mit einer Nebenreaktion einher: Nach etwa 2 Min. kam es zur Ausfällung von $\mathrm{NaCl}$, wahrscheinlich durch Bildung eines Äthers (Dichlormethyl-methyläther?). Die Reaktion trat ebenfalls im gleichbehandelten Leerwert auf. $\mathrm{Da}$ das in Lösung befindliche Na-Methylat noch weitgehend überwog, wurde unter den angegebenen Bedingungen die Umesterung nicht beeinflußt. Trotz dieser Nebenreaktion hat sich Chloroform bewährt, besonders für die Cholesterinesterumesterung. Andere Lösungsmittel, z. B. Petroläther und Diäthyläther, erwiesen sich als ungünstig. Die Methode wurde ausschließlich zur Umesterung langkettiger Fettsäuren von $C_{14}$ bis $C_{22}$ angewendet. Deshalb soll darauf hingewiesen werden, $\mathrm{da} \beta$ ein Dichlormethyl-methyläther die gaschromatographische 
Tab. 2

Abhängigkeit der Umesterung der einzelnen Lipoidklassen von Alkoholatkonzentration, Substanzmenge, Zeit und Temperatur

\begin{tabular}{|c|c|c|c|c|}
\hline \multirow[t]{2}{*}{$\begin{array}{l}\text { Na-Methylat- } \\
\text { konzentration }\end{array}$} & \multirow[t]{2}{*}{ Stoffklasse } & \multirow{2}{*}{$\begin{array}{l}\text { Substanz- } \\
\text { menge } \\
\text { mg }\end{array}$} & \multicolumn{2}{|c|}{$\begin{array}{l}\text { Zeit der vollständigen Umesterung der } \\
\text { Substanz nach ihrer Lösung in } 0,4 \mathrm{~m} / \text { Chloro- } \\
\text { form und Zugabe von } 4 \mathrm{~m} / \mathrm{Na} \text {-Methylat }\end{array}$} \\
\hline & & & $\begin{array}{l}\text { Zimmertemperatur } \\
\text { Min. }\end{array}$ & $\begin{array}{l}\text { Kochen } \\
\text { Min. }\end{array}$ \\
\hline \multirow{4}{*}{$0,25 n$} & TG & 10 & 1 & 1 \\
\hline & & 20 & 1 & 1 \\
\hline & GP & 10 & 2 & 1 \\
\hline & & 20 & 2 & 1 \\
\hline \multirow{7}{*}{$0,5 n$} & TG & 10 & 1 & 1 \\
\hline &. & 40 & 2 & 1 \\
\hline & GP & 10 & 2 & 1 \\
\hline & & 40 & 2 & 1 \\
\hline & $\mathrm{CE}$ & 10 & $\emptyset$ & 5 (B) \\
\hline & & 20 & $\emptyset$ & $\emptyset$ \\
\hline & SL & 10 & $\emptyset$ & 5 \\
\hline \multirow{6}{*}{$1,0 n$} & TG & 50 & 2 (B) & 2 (B) \\
\hline & SL & 10 & 5 & 2 \\
\hline & GP & 40 & 2 (B) & 2 (B) \\
\hline & $\mathrm{CE}$ & 10 & $\emptyset$ & 2 \\
\hline & & 20 & $\emptyset$ & 5 (B) \\
\hline & & 40 & $\emptyset$ & $\emptyset$ \\
\hline
\end{tabular}

Abkilrzungen: TG = Erdnußöl-Triglyceride, $\mathrm{GP}=$ Glycerinphosphatide (Leber), $\mathrm{CE}=$ Cholesterinester (Cholesterinpalmitat) SL $=$ Serumlipoide. $\emptyset=$ keine $\cdot$ vollständige Umsetzung nach 5 Minuten. $B=$ in zusätzlichen Parallelversuchen wurde anstatt Chloroform Benzol verwendet.

Anmerkung: Bei der Umesterung von $20 \mathrm{mg} \mathrm{CE}$, gelöst in Benzol, wurden geringe Mengen (unter 5\%) nicht umgesetzt.

Analyse kurzkettiger Fettsäuren stören könnte, ähnlich $\operatorname{dem} \beta, \beta^{\prime}$-Dichlordiäthyläther bei der Analyse kurzkettiger Fettsäuren als 2-Chloräthanolester (2).

Die Methylesterextrakte wurden dünnschichtchromatographisch auf die Anwesenheit von freien Fettsäuren untersucht. Es zeigte sich, daß die Bildung von Seifen während der alkali-katalysierten Umesterung von Alkoholatkonzentration und Temperatur abhängig ist. Die Umsetzung mit $0,25 n$ und $0,5 n \mathrm{Na}$-Methylat gingen bei Zimmertemperatur praktisch ohne Bildung von Seifen vor sich (unter $1 \%$ ). In der Hitze traten mit $0,5 n$ Na-Methylat $1-3 \%$, mit $1 n$ Na-Methylat $5-10 \%$
Seifen auf. Aus diesem Grunde wurde die MethylatKonzentration in der ersten Methode nicht über 0,5n erhöht.

Die Extraktion der beschriebenen Methoden wurde überprüft: Nach Umesterung von $60,1 \mathrm{mg}$ ErdnußTriglycerid wurden $60,8 \mathrm{mg}$ Methylester zurückerhalten. Bei der komplexen Zusammensetzung der Fettsäuren ist das Triglycerid-Methylesterverhältnis $1: 1$. Somit ergab sich eine Rückgewinnung von $100 \%$.

Herm Dipl.-Chem. Dr. H. Betzing, Firma A. Nattermann \& Cie., Köln-Braunsfeld, der die zweite Methode an seinem Untersuchungsmaterial testete, danken wir für wertvolle Hinweise.

\section{Literatur}

1. Stoffel, W., F. Chu und E. H. Ahrens, Jr., Analytic. Chem. 31, 307 (1959). - 2. Oetre, K. und E. H. Ahrens, Jr., Analytic. Chem. 33, 1847 (1961). - 3. Schienk, H. und J. L. GeluermanN, Analytic. Chem. 32, 1412 (1960). - 4. Metcalfe, L. D. und A. A. Schimitz, Analytic. Chem. 33, 363 (1961). - 5. Klenk, E. und F. RennKamp, Hoppe-Seyler's Z. physiol. Chem. 267, 145 (1940). - 6. Kuemmed, D. F., J. Amer. Oil Chemists' Soc. 35; 41 (1958). - 7. LuDDX, F. E., R. A. BARFord und R. W. RIEMENsCHNEIDER, J. Amer. Oil Chemists' Soc. 37, 447 (1960). - 8.
Kaufmand, H. P. und G. Manked, Fette Seifen einschl. Anstrichmittel 65, 179 (1963). - 9. Chevallier, F. und D. Mathe, Bull. Soc. Chim. biol. 46, 509 (1964). - 10. Klenk, E. und M. Doss, in Vorbereitung. - 11. Goldrick, B. und J. HirsCH, J. Lipid Res. 4, 482 (1963). - 12. TsснӧPE, G., diese Z. 1, 167 (1963). 13. STraktu, E., Dünñschicht-Chromatographịe, Springer-Verlag, Berlin-Göttingen-Heidelberg (1962). - 14. Horning, E. C., E. H. Ahrens, Jr., S. R. Lipsky, F. H. Mattson, J. F. Mead, D. A. TurneR und W. H. Goldwater, J. Lipid Res. 5, 20 (1964).
Dr. med. Manfred Doss, Medizinische Klinik der Universität Marburg, Marburg, Mannkopffstraße 1
Dr. med. Kurt Oette, Physiologisch-Chemisches Institut der Universität Köln, 5 Köln-Lindenthal, Joseph-Stelzmann-Str. 52 